\title{
Syntenic Relationships between Genomic Profiles of Fiber-Induced Murine and Human Malignant Mesothelioma
}

\author{
Didier Jean, ${ }^{* \dagger}$ Emilie Thomas, ${ }^{\ddagger}$ Elodie Manié,,${ }^{\S \uparrow}$ \\ Annie Renier, ${ }^{* \dagger}$ Aurélien de Reynies, ${ }^{\ddagger}$ \\ Céline Lecomte," Pascal Andujar, ${ }^{* * \dagger+\ddagger \ddagger}$ \\ Jocelyne Fleury-Feith, \$§१ף \\ Françoise Galateau-Sallé, ||||$* \star *$ \\ Marco Giovannini, ${ }^{+\dagger \dagger}$ Jessica Zucman-Rossi, ${ }^{* \dagger}$ \\ Marc-Henri Stern, ${ }^{\S \uparrow}$ and Marie-Claude Jaurand ${ }^{\star \dagger}$ \\ From INSERM, U674," Paris, France; the Universite Paris \\ Descartes, ${ }^{\dagger}$ UMR-S674, Paris, France; the Ligue Nationale Contre \\ le Cancer, ${ }^{\ddagger}$ Paris, France; INSERM, U830, ${ }^{\S}$ Paris, France; Institut \\ Curie, ${ }^{\text {II }}$ Centre de Recherche, Paris, France; the Laboratoire \\ d'Ecotoxicology," Université du Havre, UFR de Sciences et \\ Techniques, UPRES-EA3222, Le Havre, France; the Service de \\ Pneumologie et de Pathologie Professionnelle, ${ }^{* * *}$ Hôpital \\ Intercommunal de Créteil, Créteil, France; INSERM, U955, \\ Equipe 4, IMRB, Créteil, France; the Faculté de Médecine, \\ Université Paris-Est, Créteil, France; the Service d'Histologie et de \\ Biologie Tumorale, ${ }^{\circledR P}$ AP - , GHU Est, Hôpital Tenon, Paris, \\ France; the Université Pierre et Marie Curie, "वाा Paris, France; \\ Service d'Anatomie Pathologique, IIII Hôpital de la Côte de Nacre, \\ Caen, France; INSERM, ERI3, *** Caen, France; and the House \\ Ear Institute, ${ }^{\mathrm{tt}}$ Center for Neural Tumor Research, Los Angeles, \\ California
}

Malignant mesothelioma (MM) is an aggressive tumor with a poor prognosis mainly linked to past asbestos exposure. Murine models of MM based on fiber exposure have been developed to elucidate the mechanism of mesothelioma formation. Genomic alterations in murine MM have now been partially characterized. To gain insight into the pathophysiology of mesothelioma, 16 murine and 35 human mesotheliomas were characterized by array-comparative genomic hybridization and were screened for common genomic alterations. Alteration of the 9p21 human region, often by biallelic deletion, was the most frequent alteration in both species, in agreement with the $C D K N 2 A / C D K N 2 B$ locus deletion in human disease and murine models. Other shared aberrations were losses of 1 p36.3-p35 and 13q14-q33 and gains of 5p15.3-p13 regions. However, some differences were noted, such as absence of recurrent altera- tions in mouse regions corresponding to human chromosome 22. Comparison between altered recurrent regions in asbestos-exposed and non-asbestos-exposed patients showed a significant difference in the 14q11.2q21 region, which was also lost in fiber-induced murine mesothelioma. A correlation was also demonstrated between genomic instability and tumorigenicity of human mesothelioma xenografts in nude mice. Overall, these data show similarities between murine and human disease, and contribute to the understanding of the influence of fibers in the pathogenesis of mesothelioma and validation of the murine model for preclinical testing. ( $A m \mathrm{~J}$ Pathol 2011, 178:881-894; DOI: 10.1016/j.ajpath.2010.10.039)

Malignant mesothelioma (MM) is a severe primary neoplastic disease. Its frequency has dramatically increased in recent decades because of occupational exposure to asbestos fibers and the long latency period between first exposure and diagnosis, ranging from 20 to more than 40 years. ${ }^{1,2}$ The growing frequency of mesothelioma was also aggravated by the delayed recognition of asbestos diseases and implementation of asbestos regulations. Despite recent epidemiological data suggesting that the peak of MM incidence may occur sooner than expected, this malignancy remains a major concern in view of the poor results of treatment and reports of mesotheliomas with no known exposure to asbestos in $20 \%$ to $40 \%$ of cases. ${ }^{3-6}$ This raises the question of the role of very low levels of asbestos, as described in the context of environmental exposure or

Supported by the Ligue Nationale Contre le Cancer: Carte d'Identité des Tumeurs (CIT) CIT1 and CIT2 programs; Ministère de l'Emploi, de la Solidarité n¹D004C; de l'Environnement $n^{\circ} \mathrm{AC008B}$; Ligue Nationale Contre le Cancer (comité de l'Oise, France); Agence Nationale de la Recherche 059 31/ANR 05; and Agence Française de Sécurité Sanitaire de l'Environnement et du Travail RD-2004-015.

Accepted for publication October 21, 2010.

Supplemental material for this article can be found at $h$ ttp://ajp. amjpathol.org or at doi:10.1016/j.ajpath.2010.10.039.

Address reprint requests to Marie-Claude Jaurand, Ph.D., INSERM, U674, 27, rue Juliette Dodu, Paris, F-75010, France. E-mail: marieclaude.jaurand@inserm.fr. 
other, as yet unidentified, risk factors. ${ }^{7-11}$ New manufactured fiber-shaped products may also be of concern, as recent studies have demonstrated that carbon nanotubes can reach subpleural tissue in mice ${ }^{12,13}$ and induce inflammation after injection in the peritoneal cavity of mice. ${ }^{14} \mathrm{Me}$ sotheliomas also occur in genetically modified cancer-sensitive mice and in conventional Fisher 344 rats exposed to carbon nanotubes by intraperitoneal and intrascrotal administration, respectively. ${ }^{15,16}$

To improve the outcome of MM, a better knowledge of the somatic alterations in neoplastic cells is necessary to improve diagnosis, treatment, and prognosis. Several techniques are available to identify MM genomic alterations, such as cytogenetics, comparative genomic hybridization $(\mathrm{CGH})$, and single nucleotide polymorphism array, or gene mutations by DNA sequencing. Data from the literature show that most human MM cases exhibit complex patterns of cytogenetic changes, with chromosomal losses being more frequent than gains, and numerous partial or total chromosome losses have been shown to be recurrent. ${ }^{17-26}$ Somatic molecular abnormalities affecting tumor suppressor genes (TSGs) have also been described in human MM. They mainly consist of codeletion of p16/CDKN2A, p14/ $A R F$, and $p 15 / C D K N 2 B$ genes, and mutations of the NF2 gene in approximately one half of cases. In contrast, the TP53 TSG is less frequently inactivated. ${ }^{27-30}$

Murine models of mesothelioma have been recently developed in wild-type (WT) and hemizygous $\mathrm{Nf2}^{+/-}$ mice after asbestos exposure by intraperitoneal inoculation, offering an unique opportunity to more clearly characterize the genomic changes caused by asbestos on mesothelial cells. ${ }^{31-33}$ These animals developed mesotheliomas 9 to 24 months after exposure, with frequent occurrence of ascitic fluid. $N f 2$ hemizygosity resulted in a higher rate of mesothelioma when compared to WT mice, with no significant difference in terms of time to tumor occurrence. ${ }^{31}$ Mesothelioma cells isolated from tumor ascites obtained from these mice displayed similar gene mutations as human $\mathrm{MM}$, characterized by frequent $\mathrm{LOH}$ at the $\mathrm{Nf} 2$ locus in $\mathrm{Nf}^{+/-}$mice and, in all genetic backgrounds, frequent inactivation of p16/Cdkn2a, p19/ Arf, p15/Cdkn2b TSGs and infrequent inactivation of Trp53. ${ }^{32,34,35}$ Only limited data on global analysis of genomic alterations have been reported to date in rodents. Only recurrent alterations in chromosome 4 were described in murine mesothelioma cells. ${ }^{36}$ A CGH study was also performed in rats after intraperitoneal administration of iron saccharate. ${ }^{37}$

The present study was designed to investigate genomic alterations in MM by comparing the genomic profile of human $\mathrm{MM}$ cases to that of fiber-induced murine $\mathrm{MM}$ assessed by array-comparative genomic hybridization $(\mathrm{aCGH})$. Genomic profiles showed numerous alterations in both species with 15 similar regions of chromosome imbalance. In human MM cells, a link was found between genomic instability and the ability of MM xenografts to develop subcutaneous tumors in immunocompromised mice. Our data also suggest that loss of human 14q11.2-q21 region is related to asbestos exposure.

These results suggest that genes in altered regions may be damaged or deregulated by asbestos exposure, and may subsequently sustain carcinogenesis. Research is currently underway to identify the genes, gene families, and pathways involved in mesothelial carcinogenesis.

\section{Materials and Methods}

\section{Mesothelioma Cells}

Murine mesothelioma cells were obtained from previous experiments in which mineral fibers, asbestos, and carcinogenic refractory ceramic fibers were inoculated into the peritoneal cavity of WT and $\mathrm{NF}^{+/-}$mice. This strategy allowed the generation of mesotheliomas mimicking the morphology and histopathology of the corresponding human cancer. ${ }^{31,35}$ Sixteen cell cultures were established from tumor ascites obtained from $12 \mathrm{Nf2}^{+/-}$mice and four WT mice, as reported elsewhere. ${ }^{31}$ The mesothelial origin was assessed by immunocytochemistry using cytokeratin and vimentin antibodies according to a previously described method. ${ }^{31}$ Histological subtypes were epithelioid (15.4\%), sarcomatoid (30.8\%), and mixed (53.8\%) MMs. Histopathological features of murine mesotheliomas were independent of the fiber type, asbestos, or refractory ceramic fibers. The genetic background did not influence tumor morphology or genetic alterations, apart from more frequent $\mathrm{LOH}$ at the NF2 locus in $\mathrm{N} f 2^{+/-}$mice. ${ }^{34}$

A total of 35 human cell cultures were obtained from confirmed malignant mesothelioma case patients: 29 male $(82.8 \%)$ and six female (17.2\%), with a mean ( \pm SD) age of 63 \pm 11 years and $69 \pm 5$ years, respectively. The study was approved by the local Ethics Committee, and human cells were obtained with the informed consent of the patients. Detailed information about the tumors was obtained from pathology reports. Most cases were epithelioid subtypes (80\%). Sarcomatoid and mixed subtypes of MM were observed in 6.7\% and $13.3 \%$ of cases, respectively. Patients' asbestos exposure was estimated by interviewer-administered questionnaire. This questionnaire comprised complete job history, including past occupational, domestic, and environmental exposures to asbestos. It was completed by face-to-face interview. ${ }^{38}$ In this series, asbestos exposure was ascertained in $24 \mathrm{MM}$ cases, in two cases that involved possible exposure, and no exposure was found in eight cases. In one case, no data were available on asbestos history.

\section{DNA Extraction and Qualification}

Cells were grown in RPMl 1640 medium with Glutamax and 25 $\mathrm{mmo} / \mathrm{L}$ HEPES, supplemented with $8 \%$ fetal calf serum, 50 $\mathrm{IU} / \mathrm{ml}$ penicillin, and $50 \mathrm{mg} / \mathrm{ml}$ streptomycin (Invitrogen, Cergy Pontoise, France). All genetic analyses were carried out with cultures of less than 10 passages. Genomic DNA was extracted as previously described, ${ }^{34}$ quantified by absorption measurement, and qualified by deposition of $100 \mathrm{ng}$ on a $0.8 \%$ agarose gel. DNA ladder was $\lambda$ Hindlll (Invitrogen).

\section{Human and Murine aCGH}

The human genome-wide CIT-CGH array (V6) contained 5822 BAC clones, with a higher coverage in genomic re- 
gions known to contain genes involved in cancer. This array was developed by partnership among the Ligue Nationale Contre le Cancer, the Genoscope, the Curie Institute, and Integragen. The genome-wide CIT M3 Mus musculus $1 \mathrm{~K}$ BAC CGH array containing 958 unique BAC clones was manufactured by the Curie Institute (GEO record: GPL3972). Hybridizations and analyses were performed as previously reported. ${ }^{39,40}$ Fluorescent signals were detected with GenePix 4000B scanner (Molecular Devices, Union City, CA) and analyzed with GenePix Pro 5.1 software. Normalization was performed by the MANOR routine ${ }^{41}$ and data were visualized on VAMP interface. ${ }^{42} \mathrm{~A}$ syntenic conversion tool was developed to visualize the "humanized" profile of murine tumors and to facilitate comparison with human profiles. This tool attributes human syntenic coordinates obtained from the University of California, Santa Cruz, Genome Bioinformatics Group to each murine BAC of the array. Gains and losses were defined by the GLAD algorithm. ${ }^{43}$ Amplifications and biallelic deletions were defined by a normalized fluorescence ratio greater than 3 and less than 0.5 , respectively. Recurrent regions of chromosomal alteration were defined as regions that encompassed at least two adjacent probes that were both gained or lost.

\section{Gene Alteration Analysis}

Gene mutations and deletions were analyzed from genomic DNA extracted from all murine and human MM subjects. PCR amplifications and sequencing were carried out on a GeneAmp 9700 apparatus (Perkin Elmer, Courtaboeuf, France) and ABI PRISM 3100 Genetic Analyzer (Applied Biosystems, Courtaboeuf, France), respectively, according to previously published methods. ${ }^{34,35}$ Analyses concerned human genes TP53, $C D K N 2 A, C D K N 2 B$, and NF2, and the murine orthologs Trp53, Cdkn2a, Cdkn2b, and Nf2.

\section{Human MM Cell Xenografts}

Female athymic BALB/c nude mice (6-8 weeks old) were purchased from Charles River Laboratories (Les Oncins, Saint Germain sur l'Arbresle, France) and maintained under pathogen-free conditions. Animal care and experimentation were conducted in compliance with institutional guidelines in France. Human MM cells were harvested using a $0.25 \%$ trypsin, $0.2 \%$ EDTA solution (w/v) (Invitrogen). Three million cells with more than $90 \%$ viability were suspended in a volume of $200 \mu \mathrm{L}$ PBS and injected subcutaneously into the scapular region of athymic nude mice. At least four mice were inoculated for each MM, and subcutaneous tumor growth was monitored twice a week.

\section{Statistical Analyses}

Statistical analyses were performed using Prism software, version 4.0c (GraphPad Software, San Diego, CA). A $t$-test was used to analyze the association between tumorigenic phenotype and number of chromosomal breakpoints. Statistical differences in the frequency of gene mutations between human and murine MMs and comparison between the occurrence of recurrent alterations in chromosomal region and tumorigenic phenotype or asbestos exposure were assessed by Fisher's exact test.

\section{Results}

\section{Genomic Characterization of Murine Mesotheliomas}

The molecular profile of murine mesotheliomas was investigated in a series of 16 murine mesotheliomas. An overview of chromosome alteration patterns is presented in Figure 1. This analysis revealed numerous genomic alterations, with an average of 10.7 (range, 4-18) altered autosomes per tumor. Interestingly, these alterations were mostly gains or

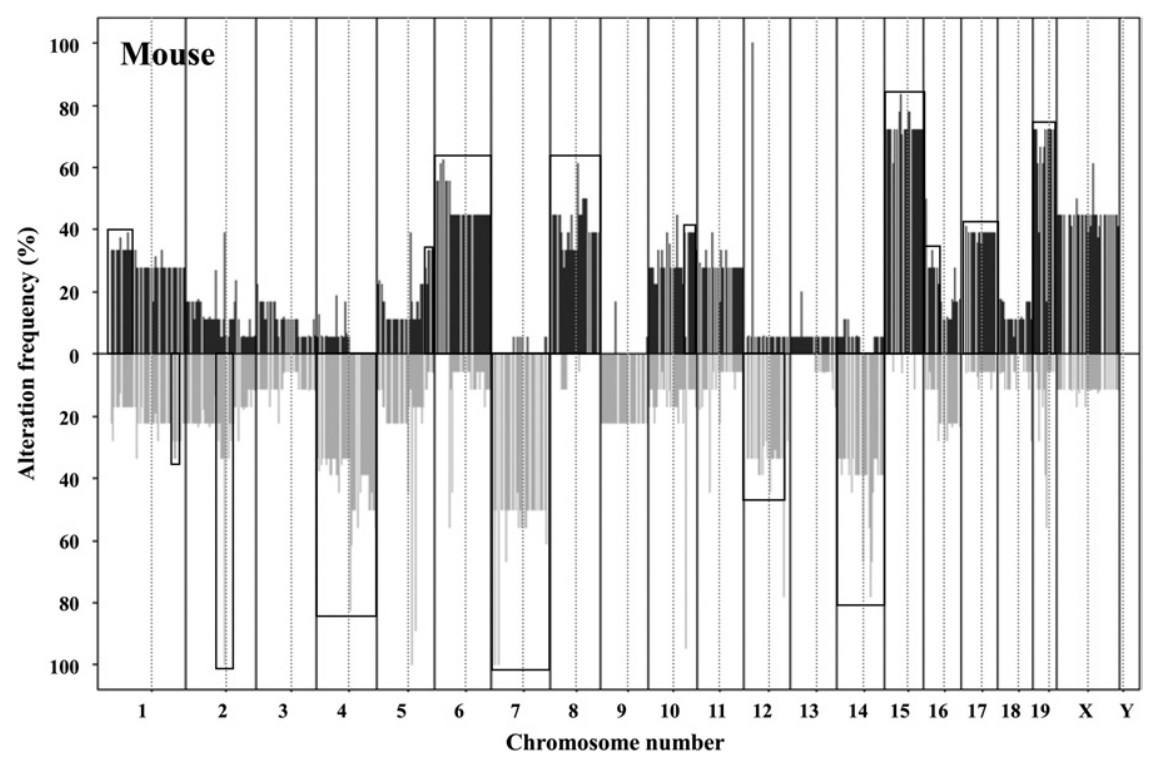

Figure 1. Summary of genomic profiles in murine mesothelioma cells. Frequency plots of gains and losses of each chromosomal region in murine MM. Bars correspond to the percentage of samples with gain or loss in a given chromosomal region. Chromosome borders are indicated by solid vertical lines and centromere positions by dashed lines. Recurrent regions of chromosomal alteration are framed with a fine line. 
Table 1. Recurrent Regions of Chromosomal Alterations (Frequency Greater than 30\%) in Murine Mesothelioma Cells

\begin{tabular}{|c|c|c|c|c|c|}
\hline Alteration & Chromosomal region & Maximal frequency (\%) & Start & End & Size (Mb) \\
\hline \multirow[t]{3}{*}{ Gain } & $\begin{array}{c}\text { 1qA1-qC1.3 } \\
\text { 5qG1.3-qG3 } \\
\text { Chr6* } \\
\text { Chr8* }^{*}\end{array}$ & $\begin{array}{l}31 \\
38 \\
56 \\
50\end{array}$ & $\begin{array}{r}0 \\
124245465\end{array}$ & $\begin{array}{r}65343589 \\
152000000\end{array}$ & $\begin{array}{l}65 \\
28\end{array}$ \\
\hline & $\begin{array}{c}\text { 10qC2-qD3 } \\
\text { Chr15* }\end{array}$ & $\begin{array}{l}38 \\
81\end{array}$ & 92606277 & 130000000 & 37 \\
\hline & $\begin{array}{c}\text { 16qA1-qB3 } \\
\text { Chr17* } \\
\text { Chr19* }\end{array}$ & $\begin{array}{l}31 \\
38 \\
75\end{array}$ & 0 & 37335257 & 37 \\
\hline \multirow[t]{2}{*}{ Loss } & $\begin{array}{c}1 \mathrm{qH} 2.1-\mathrm{qH} 5 \\
2 \mathrm{qE} 1-\mathrm{qE} 3 \\
\mathrm{Chr}^{*}\end{array}$ & $\begin{array}{l}38 \\
31 \\
81\end{array}$ & $\begin{array}{r}158533294 \\
88372743\end{array}$ & $\begin{array}{l}188288765 \\
113343671\end{array}$ & $\begin{array}{l}30 \\
25\end{array}$ \\
\hline & $\begin{array}{c}\text { Chr7* }^{*} \\
\text { 12qA1.1-qE } \\
\text { Chr14* }^{*}\end{array}$ & $\begin{array}{l}63 \\
44 \\
69\end{array}$ & 0 & 105426793 & 105 \\
\hline
\end{tabular}

${ }^{*}$ Several gained or lost regions encompassed whole chromosomes in some murine MMs (see Supplemental Figure S1, A-C, for details at http://ajp.amjpathol.org).

losses of entire chromosomes. Recurrent regions of chromosomal alterations were then defined as regions with at least two adjacent probes gained or lost at a frequency greater than 30\% (Table 1). Frequent recurrent gained regions (more than $50 \%$ of cases) included chr 15 ( $75 \%$ to $81 \%), 19 q C 3-q D 2$ (75\%), 19qA-qC1 (63\% to $75 \%), 6 q A 1-$ $\mathrm{qB} 1(50 \%$ to $56 \%)$, and $8 \mathrm{qC} 2-\mathrm{qC} 5(50 \%)$, and frequent losses included regions in 4qC4-qD1 (50\% to $81 \%$ ), 14qD3-qE2.1 (50\% to $69 \%$ ), 4qD3-qE2.1 (50\% to $63 \%$ ), chr7 (56\% to $63 \%$ ), and $14 \mathrm{qB}$ (50\%) (see Supplemental Figure S1, A-C, at http://ajp.amjpathol.org). Minimal regions of chromosomal loss were then delineated on the basis of the alteration frequency and the presence of an interstitial deletion in at least two MMs to identify potential tumor suppressor genes and the miRNAs lost in murine mesothelioma cells (Table 2 and Supplemental Figure S1, A-C at $h$ ttp:// ajp.amjpathol.org). The most remarkable characteristic was the high frequency of interstitial deletion in chromosome 4 $(81 \%)$, which was biallelic in six tumors (Table 2). The 5-Mb minimal region of this recurrent deletion located in $4 q C 4$ contained the Cdkn2a/Cdkn2b gene locus. Another recurrent interstitial deletion was observed on chromosome 14 (14qE2.1) in 11 of $16 \mathrm{MM}$ cells, containing the Diap3, Pcdh9, and Pcdh2O candidate genes. Deletion of the Nf2 region in 11qA1 was infrequent (3/16) and found only in a $\mathrm{Nf}^{+/-}$ background. Three high-level gained regions containing the proto-oncogene $M y c$ and several cadherins were identified. Four regions of homozygous deletions were observed, including Sav1, the putative TSG involved in the Hippo signaling pathway, as candidate target gene (Table 2).

\section{Genomic Characterization of Human Mesotheliomas}

The genomic characterization of a series of 35 human MMs also demonstrated a high rate of rearrangements

Table 2. Minimal Regions of Chromosomal Imbalance Based on Alteration Frequency and Occurrence of Interstitial Deletions, Amplifications, and Biallelic Deletions in 16 Murine Mesothelioma Cells

\begin{tabular}{|c|c|c|c|c|c|c|c|c|}
\hline Alteration & $\begin{array}{c}\text { Chromosomal } \\
\text { region }\end{array}$ & $\begin{array}{l}\text { Number of } \\
\text { MMs (\%) }\end{array}$ & Start & End & $\begin{array}{l}\text { Size } \\
(\mathrm{Mb})\end{array}$ & $\begin{array}{c}\text { Gene } \\
\text { number }\end{array}$ & Gene & miRNA \\
\hline \multirow[t]{4}{*}{$\begin{array}{l}\text { Minimal regions } \\
\text { of deletion }\end{array}$} & $1 \mathrm{qH} 2.2-\mathrm{qH} 2.3$ & $6(38)$ & 162074549 & 171028275 & 9 & $>10$ & $\begin{array}{l}\text { Gas5, } R x r g \\
\quad P b \times 1\end{array}$ & $\begin{array}{l}\text { Mir214, } \\
\text { Mir2134-1, } \\
\text { Mir689-1 }\end{array}$ \\
\hline & $4 q C 4$ & $13(81)$ & 84800547 & 89483516 & 5 & $>10$ & Cdkn2a, Cdkn2b & $\begin{array}{l}\text { Mir491, } \\
\text { Mir31 }\end{array}$ \\
\hline & $\begin{array}{c}4 q C 7 \\
12 q C 1-q C 2\end{array}$ & $\begin{array}{l}9(56) \\
7(44)\end{array}$ & $\begin{array}{r}102961540 \\
60575140\end{array}$ & $\begin{array}{r}114389001 \\
71056044\end{array}$ & $\begin{array}{l}11 \\
10 \\
5\end{array}$ & $\begin{array}{l}>10 \\
>10\end{array}$ & $\begin{array}{l}\text { Cdkn2c, Faf1 } \\
\text { Sav1 }\end{array}$ & $\begin{array}{l}\text { Mir761 } \\
\text { Mir681 }\end{array}$ \\
\hline & $\begin{array}{l}14 q \mathrm{qB} \\
14 \mathrm{qE} 2.1\end{array}$ & $\begin{array}{r}8(50) \\
11(69)\end{array}$ & $\begin{array}{l}39021984 \\
85113991\end{array}$ & $\begin{array}{l}43790944 \\
96312501\end{array}$ & $\begin{array}{r}5 \\
11\end{array}$ & $\begin{array}{r}>10 \\
5\end{array}$ & $\begin{array}{l}\text { Diap3, } \overline{T d r d 3}, \\
\text { Pcdh20, } \\
\text { Pcdh9, Klh/1 }\end{array}$ & - \\
\hline \multirow[t]{3}{*}{ Amplification } & $8 q A 1.1$ & $1(6)$ & 0 & 23875369 & 24 & $>10$ & Elavl1, Gas6 & $\begin{array}{l}\text { Mir1968, } \\
\text { Mir2144 }\end{array}$ \\
\hline & 8qC5-qD1 & $1(6)$ & 91443012 & 107427526 & 16 & $>10$ & $\begin{array}{l}\text { Mmp2, Mmp15, } \\
\text { Cdh11, Cdh5, } \\
\text { Cdh8 }\end{array}$ & Mir138-2 \\
\hline & 15qD1 & $1(6)$ & 58895730 & 66093803 & 7 & $>10$ & Myc & - \\
\hline \multirow{3}{*}{ Biallelic deletion } & $1 q A 1$ & $1(6)$ & 0 & 10359697 & 10 & $>10$ & - & - \\
\hline & 4qC4 & $6(38)$ & 84800547 & 89483516 & $\begin{array}{r}5 \\
10\end{array}$ & $>10$ & Cdkn2a, Cdkn2b & Mir31 \\
\hline & $14 q B$ & $1(6)$ & 39021984 & 43790944 & 5 & $>10$ & savi - & - \\
\hline
\end{tabular}

Genes clearly or potentially involved in oncogenesis are indicated in bold. 


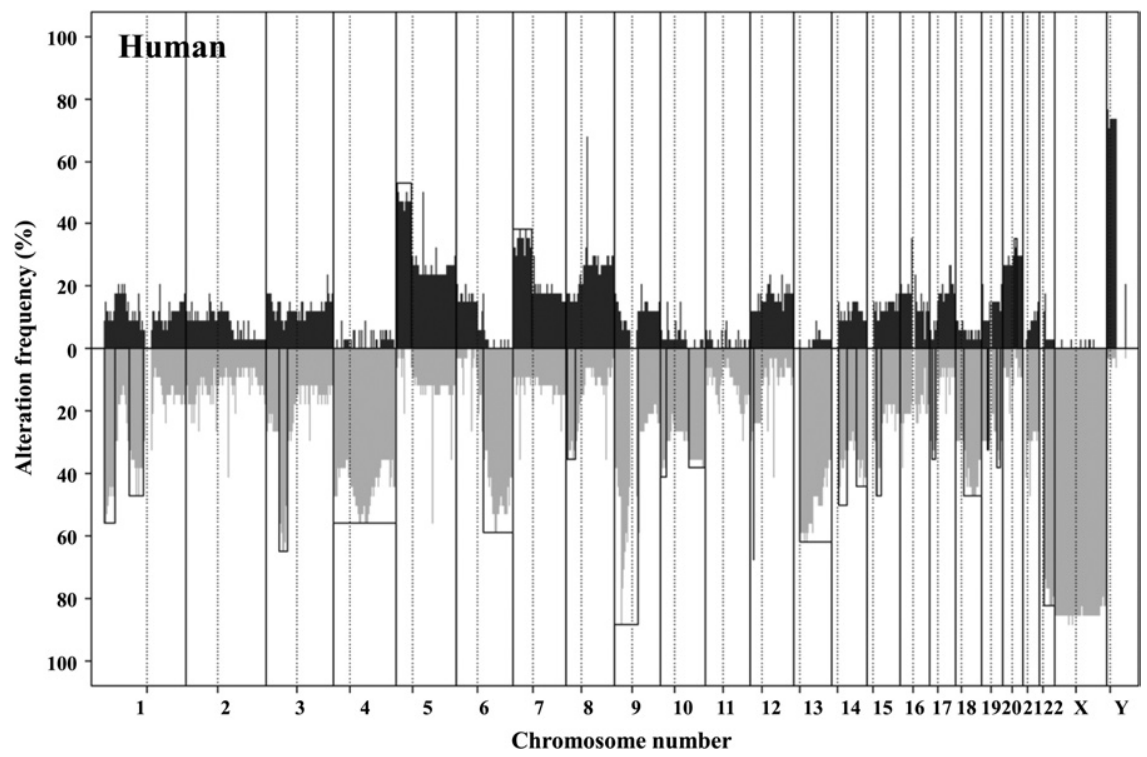

Figure 2. Summary of genomic profiles in human mesothelioma cells. Frequency plots of gains and losses of each chromosomal region in human MM. Bars correspond to the percentage of samples with gain or loss in a given chromosomal region. Chromosome borders are indicated by solid vertical lines and centromere positions by dashed lines. Recurrent regions of chromosomal alteration are framed with a fine line.

(Figure 2). Two tumors displayed up to 21 altered autosomes (mean of 13.8 altered autosomes per tumor; range: 1-21). However, most alterations did not involve entire chromosomes, and monoallelic gains were less frequent than in murine MMs. Nine recurrent regions of loss with a frequency greater than $50 \%$ were observed: 9p22-p13 (51\% to 91\%), 22q (57\% to 80\%), 3p22-p14 (51\% to 63\%), 13q11-q21 (51\% to 60\%), 6q21-q22 (51\% to 57\%), 4q21-q24 (51\%to 54\%), 12p13 (54\%), 1 p36.3 (51\%), and $6 q 24-q 25(51 \%)$, and only one region of gain was observed: 5p15.3 (51\%) (see Supplemental Figure S2 at http://ajp.amjpathol.org). Recurrent regions of chromosomal alterations with a frequency greater than $30 \%$ are shown in Table 3. Ten high-level gained regions and 12 regions containing homozygous deletions were detected (Table 4). Homozygous deletions in 9p21 (22/
35), 22q11.2 (3/35), 1p21 (2/35), and 9p23 (2/35) were identified in several human MMs, but none of the amplicons were recurrent.

\section{Humanized Murine aCGH}

Syntenic analysis of the murine genomic profiles revealed similarities between their humanized profiles and the genomic profiles obtained from the human series of MM. In particular, 12 regions recurrently altered (frequency greater than $30 \%$ ) in both the human disease and the murine model were identified (Table 5). Human and murine MM cells shared the same lost regions with the highest frequency: Hs (Homo sapiens) 9p24-p13 (91\%) and Mm (Mus musculus) 4qC3-C5 (81\%). Other altered regions, such as Hs 1p36.3-p35

Table 3. Recurrent Regions of Chromosomal Alterations (Frequency Greater than 30\%) in Human Mesothelioma Cells

\begin{tabular}{|c|c|c|c|c|c|}
\hline Alteration & Chromosomal region & Maximal frequency (\%) & Start & End & Size $(\mathrm{Mb})$ \\
\hline \multirow[t]{3}{*}{ Gain } & 5p15.3-p11 & 51 & 0 & 49464271 & 49 \\
\hline & 7p22-p11.2 & 37 & 0 & 57346208 & 57 \\
\hline & $20 q 11.2-q 13.1$ & 34 & 34168802 & 43536662 & 9 \\
\hline \multirow[t]{19}{*}{ Loss } & 1p36.3-p35 & 51 & 0 & 30383282 & 30 \\
\hline & 1p31-p12 & 40 & 75229478 & 119360570 & 43 \\
\hline & 3p23-p14 & 63 & 34981835 & 64352460 & 29 \\
\hline & Chr4 & 54 & & & \\
\hline & $6 q 14-q 27$ & 57 & 80213243 & 170629779 & 90 \\
\hline & 8p23-p12 & 31 & 1790686 & 31056165 & 29 \\
\hline & 9p24-q21 & 91 & 0 & 70025358 & 70 \\
\hline & 10p15-p12 & 37 & 0 & 21068361 & 21 \\
\hline & $10 q 23-q 26$ & 37 & 86173438 & 135071951 & 49 \\
\hline & $12 p 13$ & 54 & 10844968 & 12614264 & 2 \\
\hline & $13 q$ & 60 & & & \\
\hline & $14 q 11.2-q 21$ & 40 & 0 & 42832378 & 43 \\
\hline & 14q24-q32 & 40 & 76307162 & 106230236 & 30 \\
\hline & $15 q 13-q 21$ & 40 & 27018560 & 44046064 & 17 \\
\hline & 17p13-p11.2 & 34 & 5782075 & 17473056 & 12 \\
\hline & 18q12-q23 & 46 & 23824248 & 75939456 & 52 \\
\hline & 19p13.1-p12 & 31 & 18161120 & 24246700 & 6 \\
\hline & $19 q 13.2-q 13.4$ & 31 & 47672188 & 59385609 & 12 \\
\hline & $22 q$ & 80 & & & \\
\hline
\end{tabular}


886 Jean et al

AJP February 2011, Vol. 178, No. 2

Table 4. Amplification and Biallelic Deletions in Human Mesothelioma Cells

\begin{tabular}{|c|c|c|c|c|c|c|c|c|}
\hline Alteration & $\begin{array}{l}\text { Chromosomal } \\
\text { region }\end{array}$ & $\begin{array}{c}\text { Number of } \\
\text { MMs }\left(\mathrm{N}_{\mathrm{T}}=35\right)\end{array}$ & Start & End & $\begin{array}{l}\text { Size } \\
(\mathrm{Mb})\end{array}$ & Gene number & Gene & miRNA \\
\hline \multirow[t]{10}{*}{ Amplification } & 1p34.2-p34.1 & 1 & 40464163 & 43936177 & 3 & $>10$ & $C D C 20$ & $\begin{array}{l}\text { MIR30E, } \\
\text { MIR30C1 }\end{array}$ \\
\hline & $2 \mathrm{p} 12$ & 1 & 75046439 & 76197958 & 1 & 5 & $\begin{array}{l}\text { POLE4, TACR1, FAM176A, } \\
\text { MRPL19, C2orf3 }\end{array}$ & - \\
\hline & $4 q 31.2$ & 1 & 147916169 & 150226685 & 2 & $>10$ & - & MIR548G \\
\hline & $5 p 15.3$ & 1 & 0 & 2692035 & 3 & $>10$ & TERT & - \\
\hline & $7 \mathrm{p} 21-\mathrm{p} 15$ & 1 & 9198514 & 29922960 & 21 & $>10$ & $\begin{array}{l}\text { TWIST1, GPNMB, IL6, NPY, } \\
\text { HNRNPA2B1, HOXA9, } \\
\text { HOXA13 }\end{array}$ & $\begin{array}{l}\text { MIR1302-6, } \\
\text { MIR1183, } \\
\text { MIR148A, } \\
\text { MIR196B }\end{array}$ \\
\hline & $7 p 14$ & 1 & 39469174 & 43369192 & 4 & $>10$ & - & - \\
\hline & $9 p 24$ & 1 & 442668 & 8228482 & 8 & $>10$ & $R L N 2, K D M 4 C$ & MIR101-2 \\
\hline & $10 q 26$ & 1 & 123070217 & 126566539 & 3 & $>10$ & FGFR2 & - \\
\hline & $19 q 12-q 13.1$ & 1 & 33184604 & 38838451 & 6 & $>10$ & - & - \\
\hline & $20 q 13.3$ & 1 & 54336834 & 57746514 & 3 & $>10$ & AURKA & $\begin{array}{l}\text { MIR296, } \\
\text { MIR298 }\end{array}$ \\
\hline \multirow[t]{12}{*}{$\begin{array}{l}\text { Biallelic } \\
\quad \text { deletion }\end{array}$} & $1 \mathrm{p} 21$ & 2 & 101859311 & 105068099 & 3 & 8 & $\begin{array}{l}\text { OLFM3, COL11A1, RNPC3, } \\
\text { AMY2B, AMY2A, AMY1A, } \\
\text { AMY1B, AMY1C }\end{array}$ & - \\
\hline & $4 p 15.3$ & 1 & 12344454 & 13298102 & 1 & 3 & RAB28, NKX3-2, BOD1L & - \\
\hline & $5 q 34$ & 1 & 166908572 & 170038731 & 3 & $>10$ & - & $\begin{array}{l}\text { MIR103-1, } \\
\text { MIR103-1AS, } \\
\text { MIR218-2, } \\
\text { MIR585 }\end{array}$ \\
\hline & $9 p 23$ & 2 & 8228482 & 13419660 & 5 & 4 & $\begin{array}{l}\text { PTPRD, TYRP1, C9orf150, } \\
\text { MPDZ }\end{array}$ & - \\
\hline & $9 p 21$ & 22 & 20172510 & 22317110 & 2 & $>10$ & $C D K N 2 A, C D K N 2 B$ & $\begin{array}{l}\text { MIR31, } \\
\text { MIR491 }\end{array}$ \\
\hline & $10 q 26$ & 1 & 126566539 & 131109233 & 5 & $>10$ & - & - \\
\hline & $11 q 22-q 23$ & 1 & 106448945 & 114320480 & 8 & $>10$ & $\begin{array}{l}\text { ATM, SDHD, ZBTB16, } \\
\quad P P P 2 R 1 B\end{array}$ & $\begin{array}{l}\text { MIR34B, } \\
\text { MIR34C }\end{array}$ \\
\hline & $13 q 21$ & 1 & 65415360 & 70465908 & 5 & 3 & PCDH9, KLHL1, DACH1 & - \\
\hline & $16 \mathrm{p} 13.3$ & 1 & 5147035 & 8712134 & 4 & 4 & $\begin{array}{l}\text { A2BP1, TMEM114, } \\
\text { C16orf68, ABAT }\end{array}$ & - \\
\hline & $18 p 11.3$ & 1 & 5196459 & 7203642 & 2 & 6 & $\begin{array}{l}\text { ZFP161, EPB41L3, TTMA, } \\
\text { L3MBTL4, ARHGAP28, } \\
\text { LAMA1 }\end{array}$ & - \\
\hline & $22 q 11.2$ & 3 & 19570585 & 20084258 & 1 & 9 & $\begin{array}{l}\text { SNAP29, CRKL, AIFM3, } \\
\text { LZTR1, THAP7, P2RX6, } \\
\text { SLC7A4, GGT2, RIMBP3B }\end{array}$ & MIR649 \\
\hline & $22 q 12$ & 1 & 28155132 & 28374240 & $<1$ & 1 & $\begin{array}{l}\text { RFPL1, NEFH, THOC5, } \\
\text { NIPSNAP1, NF2 }\end{array}$ & - \\
\hline
\end{tabular}

Genes clearly or potentially involved in oncogenesis are indicated in bold.

(Mm 4qD2.3-4qE2) and Hs 13q14-q33 (Mm 14qD1qE2.3), showed a frequency of loss greater than $50 \%$. Gain in Hs 5p15.3-p13 (Mm 15qA1-qB3.1) and losses in Hs 3p21-p14 (Mn 14qA1-14qA3) and Hs 13q12 (Mn
14qC3) were also characterized as recurrent in both species. However, it must be noted that some loci within these human recurrent regions (Hs 3p21, Hs 13q13) were not altered in mice (see Supplemental

Table 5. Comparison of Chromosomal Alterations (Frequency Greater than 30\%) between Human and Murine Mesothelioma Cells

\begin{tabular}{|c|c|c|c|c|c|c|c|}
\hline \multirow[b]{2}{*}{ Alteration } & \multicolumn{5}{|c|}{ Human } & \multicolumn{2}{|c|}{ Murine } \\
\hline & $\begin{array}{c}\text { Chromosomal } \\
\text { region }\end{array}$ & $\begin{array}{c}\text { Maximal } \\
\text { frequency }(\%)\end{array}$ & Start & End & $\begin{array}{l}\text { Size } \\
(\mathrm{Mb})\end{array}$ & Chromosomal region & $\begin{array}{c}\text { Maximal } \\
\text { frequency }(\%)\end{array}$ \\
\hline \multirow[t]{3}{*}{ Gain } & 5p15.3-p13 & 49 & 7387737 & 39283396 & 32 & $15 q A 1-q B 3.1$ & 75 \\
\hline & 7p22-p21 & 37 & 188237 & 12020141 & 12 & $5 q G 2 / 6 q A 1$ & $38 / 58$ \\
\hline & 7p15-p14 & 37 & 24340863 & 30917523 & 7 & 6qB2.3-qB3 & 44 \\
\hline \multirow[t]{12}{*}{ Loss } & 1p36.3-p35 & 51 & 2058046 & 29574368 & 28 & 4qD2.3-4qE2 & 63 \\
\hline & 3p21-p14 & 60 & 52571782 & 63683864 & 11 & $14 q A 1 / 14 q A 3$ & $38 / 38$ \\
\hline & $6 q 15-q 16$ & 40 & 89148060 & 100061960 & 11 & 4qA3-qA5 & 38 \\
\hline & $8 p 21$ & 31 & 22048391 & 26316410 & 4 & $14 q D 1$ & 44 \\
\hline & $9 p 24-p 13$ & 91 & 8228482 & 36895443 & 29 & 4qA5-B1/4qC3-C5 & $44 / 81$ \\
\hline & $10 q 23$ & 34 & 86679954 & 87489928 & 1 & $14 q A 3-q B$ & 38 \\
\hline & $10 q 26$ & 37 & 121395903 & 127901902 & 7 & 7qF2-qF3 & 56 \\
\hline & $13 q 12$ & 57 & 19505755 & 19703538 & 0 & $14 q C 3$ & 44 \\
\hline & $13 q 14-q 33$ & 57 & 42908596 & 101265081 & 58 & 14qD1-qE2.3 & 69 \\
\hline & $14 q 11.2-q 21$ & 40 & 19150554 & 42832378 & 24 & $\begin{array}{l}\text { 12qB3-qC1/ } \\
14 q C 1-q C 2\end{array}$ & $44 / 44$ \\
\hline & 14q24-q32 & 37 & 77705005 & 94277131 & 17 & $12 q D 3-q E$ & 38 \\
\hline & $19 q 13.2-q 13.4$ & 31 & 50052470 & 54919450 & 5 & 7qA2/7qB3 & $56 / 56$ \\
\hline $\begin{array}{l}\text { Biallelic } \\
\text { deletion }\end{array}$ & $9 p 21$ & 63 & 20172510 & 22317110 & 2 & $4 q C 4$ & 40 \\
\hline
\end{tabular}


Table 6. Minimal Regions of Chromosomal Alterations in Human Mesothelioma Cells

\begin{tabular}{|c|c|c|c|c|c|c|c|c|}
\hline $\begin{array}{l}\text { Alteration } \\
\text { (loss) }\end{array}$ & $\begin{array}{l}\text { Chromosomal } \\
\text { region }\end{array}$ & $\begin{array}{c}\text { Maximal } \\
\text { frequency }(\%) \\
\text { (human/mouse) }\end{array}$ & Start & End & $\begin{array}{l}\text { Size } \\
(\mathrm{Mb})\end{array}$ & Gene number & Gene & miRNA \\
\hline \multirow{4}{*}{$\begin{array}{l}\text { Human/mouse } \\
\text { comparison }\end{array}$} & $1 \mathrm{p} 36.3$ & $51 / 63$ & 4356069 & 6292626 & 2 & $>10$ & CHD5 & - \\
\hline & $1 \mathrm{p} 36.1$ & $46 / 50$ & 19083345 & 20986850 & 2 & $>10$ & $N B L 1$ & MIR1290 \\
\hline & 9p21 & $91 / 81$ & 20172510 & 22317110 & 2 & $>10$ & $C D K N 2 A, C D K N 2 B$ & $\begin{array}{l}\text { MIR31, } \\
\text { MIR491 }\end{array}$ \\
\hline & $13 q 21$ & $\begin{array}{c}49 / 69 \\
\text { (human) }\end{array}$ & 62637997 & 70465908 & 8 & 3 & PCDH9, KLHL1, DACH1 & - \\
\hline \multirow[t]{13}{*}{$\begin{array}{l}\text { Interstitial } \\
\text { deletions in } \\
\text { humans }\end{array}$} & 1p22-p21 & 40 & 92918111 & 101318454 & 8 & $>10$ & - & $\begin{array}{l}\text { MIR760, } \\
\text { MIR137, } \\
\text { MIR553 }\end{array}$ \\
\hline & $3 p 21$ & 60 & 45214909 & 50409942 & 5 & $>10$ & $\begin{array}{l}\text { SETD2, MST1R, } \\
\text { PLXNB1, RASSF1, } \\
\text { RBM5, SEMA3B, } \\
\text { SEMA3F, HYAL1, } \\
\text { HYAL2 }\end{array}$ & $\begin{array}{l}\text { MIR1226, } \\
\text { MIR2115, } \\
\text { MIR711, } \\
\text { MIR425, } \\
\text { MIR191, } \\
\text { MIR566 }\end{array}$ \\
\hline & $4 p 16-p 15.3$ & 46 & 9614707 & 12344454 & 3 & 5 & $\begin{array}{l}\text { SLC2A9, WDR1, } \\
\text { ZNF518B, CLNK, } \\
\text { HS3ST1 }\end{array}$ & MIR572 \\
\hline & $4 \mathrm{q} 13$ & 49 & 63518558 & 65274228 & 2 & 1 & TECRL & - \\
\hline & $4 \mathrm{q} 22-23$ & 54 & 92471635 & 101247956 & 9 & $>10$ & $\begin{array}{r}\text { BMPR1B, } \\
\text { UNC5C }\end{array}$ & - \\
\hline & $6 q 22$ & 57 & 115807782 & 120525326 & 5 & $>10$ & - & MIR548B \\
\hline & $6 q 24-q 25$ & 51 & 144840012 & 155794638 & 11 & $>10$ & $\begin{array}{l}\text { LATS1, } \\
\quad \text { AKAP12 }\end{array}$ & - \\
\hline & 9p22 & 49 & 14010653 & 14732051 & 1 & 4 & $\begin{array}{l}\text { NFIB, ZDHHC21, CER1, } \\
\quad \text { FREM1 }\end{array}$ & - \\
\hline & $9 p 11.1$ & 43 & 42843412 & 66195312 & 23 & 4 & $\begin{array}{l}\text { ANKRD20A3, FAM75A6, } \\
\text { CNTNAP3B, FAM75A7 }\end{array}$ & - \\
\hline & 10q23-q24 & 37 & 87489928 & 108067973 & 21 & $>10$ & $\begin{array}{l}\text { BMPR1A, SNCG, PTEN, } \\
\text { FAS, LGI1, NFKB2, } \\
\text { SUFU }\end{array}$ & $\begin{array}{l}\text { MIR346, } \\
\text { MIR107, } \\
\text { MIR607, } \\
\text { MIR1287, } \\
\text { MIR608, } \\
\text { MIR146B, } \\
\text { MIR1307, } \\
\text { MIR936, } \\
\text { MIR609 }\end{array}$ \\
\hline & $12 \mathrm{p} 13$ & 54 & 11584100 & 12614264 & 1 & 6 & $\begin{array}{l}\text { ETV6, BCL2L14, LRPG, } \\
\text { MANSC1, LOH12CR1, } \\
\text { DUSP16 }\end{array}$ & MIR1244 \\
\hline & $13 q 13$ & 60 & 31067328 & 33059957 & 2 & 9 & $\begin{array}{l}\text { RXFP2, FRY, ZAR1L, } \\
\text { BRCA2, NABP2L1, } \\
\text { NABP2L2, PDS5B, KL, } \\
\text { STARD13 }\end{array}$ & - \\
\hline & $15 q 14-q 21$ & 40 & 30654132 & 44046064 & 13 & $>10$ & THBS1 & $\begin{array}{l}\text { MIR1233, } \\
\text { MIR626, } \\
\text { MIR627 }\end{array}$ \\
\hline $\begin{array}{l}\text { High frequency } \\
\text { in humans }\end{array}$ & $22 q 12-q 13$ & 80 & 32678146 & 42309461 & 10 & $>10$ & EP300, BIK & $\begin{array}{l}\text { MIR658, } \\
\text { MIR659, } \\
\text { MIR1281, } \\
\text { MIR33a }\end{array}$ \\
\hline
\end{tabular}

Genes clearly or potentially involved in oncogenesis are indicated in bold.

Figure S2, D and L, at http://ajp.amjpathol.org). Furthermore, several regions of chromosomal imbalance in human MM were not recurrently altered in the murine genomic profile. The most striking difference was the absence of recurrent alteration in mouse genomic regions corresponding to human chromosome 22. Other human regions characterized by a frequency of loss greater than $50 \%$, such as Hs $4 \mathrm{q} 21-\mathrm{q} 24$, Hs $6 \mathrm{q} 21-$ q22, Hs 6q24-q25, and Hs 12p13, were also not recurrently lost in the murine syntenic regions. Furthermore, regions of high-level gain differed between human and murine MMs, and only the frequent homozygous deletion in the Hs 9p21 region was detected in murine $\mathrm{MM}(\mathrm{Mn} 4 \mathrm{qC} 4)$.

\section{Human Candidate Genes Associated with Minimal Regions of Chromosomal Imbalance}

To determine genes or miRNAs potentially involved in human mesothelial carcinogenesis, minimal altered re- gions were defined on the basis of several criteria. First, CGH array data were compared between murine and human MMs, and four minimal regions of loss were delineated in both species: 1p36.3, 1p36.1, 9p21, and 13q21 (Table 6 and Supplemental Figure S2, A, B, I, and $\mathrm{M}$ at http://ajp.amjpathol.org). The 9p21 region, which encompassed the CDKN2A and CDKN2B genes, showed the highest alteration frequency in both species. The presence of homozygous deletions in this region was also detected in $63 \%$ and $40 \%$ of human and murine MMs, respectively (Table 5). Three other regions also contained putative TSG, such as CHD5 (1p36.3), NBL1 (1p36.1), PCDH9, and DACH1 (13q21). Interestingly, the 13q21 region, in which one of the human MMs showed a homozygous deletion (Table 4), contained only three coding genes (Table 6). Second, 13 new minimal regions of chromosomal loss in human MM were defined on the basis of the alteration frequency and the presence of an interstitial deletion in at least three MMs (Table 6 and Supplemental Figure S2 
Table 7. Percentage of Cases Showing Gene Alterations in Human and Murine Mesothelioma Cells

\begin{tabular}{lccc}
\hline Human/murine gene & $\begin{array}{c}\text { Alteration frequency } \\
\text { in humans (\%) }\end{array}$ & $\begin{array}{c}\text { Alteration frequency } \\
\text { in mice (\%) }\end{array}$ & $P$ value* \\
\hline TP53/Trp53 & 20 & 25 & 0.72 \\
NF2/Nf2 & 46 & 75 & 0.07 \\
CDKN2A/Cdkn2a & 82 & 56 & 0.09 \\
CDKN2B/Cdkn2b & 76 & 60 & 0.43 \\
\hline
\end{tabular}

*Fisher's exact test.

at http://ajp.amjpathol.org). Seven of these regions (3p21, 4q22-23, 6q24-q25, 10q23-q24, 12p13, 13q13, and $15 q 14-q 21)$ contained genes that have been previously described as being involved in cancer progression, as shown in Table 6. The other six regions (1p22-p21, 4p16-p15.3, 4q13, 6q22, 9p22, 9p11.1, and 15q14q21) did not contain any potential TSG but encompassed several miRNA loci. Third, despite the entire loss of 22q in $57 \%$ of human MMs, the smaller q12-q13 region, characterized by the highest alteration frequency $(80 \%)$, was delineated. This region contains two potential genes previously described to be involved in tumor progression (EP300, BIK) and four miRNAs (Table 6). The NF2 gene locus located in 22q12 showed an alteration frequency of 71\%(seeSupplementalFigureS2, Q, athttp://ajp.amjpathol. org). Fourth, genes and miRNAs localized in high-level gained regions and in regions with homozygous deletions were identified (Table 4). The most remarkable finding was the presence of the NF2 locus in one of the regions bearing biallelic deletion (22q12). Furthermore, several human genes possibly involved in tumor progression of MM, such as CDC20 (1p34.2-p34.1), TERT (5p15.3), FGFR2 (10q26), and AURKA (20q13.3), were localized in high-level gained regions (Table 4).

\section{Gene Mutations in Human and Murine MM}

The rates of mutation in human TP53, CDKN2A/CDKN2B, and NF2 genes and in the orthologous murine genes are reported in Table 7 , confirming previously published data, but based on a larger series. ${ }^{34,35,44}$ No significant difference was observed for the percentage of human and murine MMs showing gene alterations. Mutations in TP53 TSG were found in a fairly low percentage in both human and murine $\mathrm{MMs}$ ( $20 \%$ and $25 \%$, respectively). In contrast, CDKN2A and CDKN2B genes were altered by high frequency deletions, especially in human MM. Comparison between NF2 alterations in mice and humans is more difficult, as $75 \%$ (12/16) of murine MM cultures were derived from mesotheliomas developed in $\mathrm{Nf}^{+/-}$mice. Nevertheless, deletions were the most frequent type of mutation in both human and murine MMs $(65 \%$ and $92 \%$, respectively).

\section{Association between Genomic Alterations and Histological Subtype of MM in Mice and Humans}

Comparison of recurrent regions of chromosomal alterations with histological MM subtypes underlined differ- ent frequencies among the altered regions. As biphasic subtype is a mixed population, comparisons were made only between epithelioid and sarcomatoid subtypes. Results should be considered with caution regarding the low percentage of sarcomatoid human MM $(6.7 \%)$ and murine epithelioid MM (15.4\%). Gain in Hs 5p15.3-p11 and losses in Hs 3p23-p14. Hs 6q14q27, and Hs 10p15-p12 were observed in more than $50 \%$ and $0 \%$ of epithelioid and sarcomatoid subtypes, respectively. In mice, loss of $\mathrm{Mm} 2 \mathrm{qE} 1-\mathrm{qE} 3$ showed the same distribution. At the opposite, $\mathrm{Mm} \mathrm{1qH2.1-}$ $\mathrm{qH} 5$ and $\mathrm{Mm}$ 16qA1-qB3 alterations showed a frequency greater than $50 \%$ in sarcomatoid subtypes and $0 \%$ in epithelioid subtypes. However, there were no similarities between human and mouse MM in terms of type and location of recurrent alterations according to histological subtype.

\section{Association between Genomic Alterations and Xenograft Growth of Human MM Cells}

Tumorigenicity of 32 human MM cells was determined in immunocompromised mice. In vivo tumor growth was observed with $23 \mathrm{MMs}$. For each case, the tumorigenic potency was compared to that of genomic alterations. Several recurrent genomic alterations were more fre-

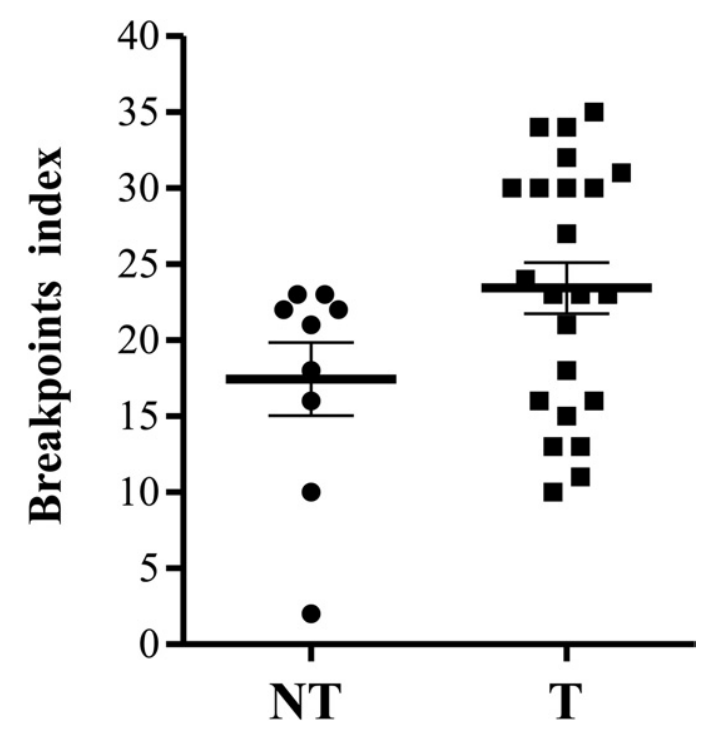

Figure 3. Genomic instability and tumorigenic potency in nude mice xenografts of human mesothelioma cells. Breakpoint index was determined for each human MM and plotted separately for nontumorigenic (NT) and tumorigenic (T) MM cells. Medians are indicated by horizontal bars. 
Human 14q11.2-q21

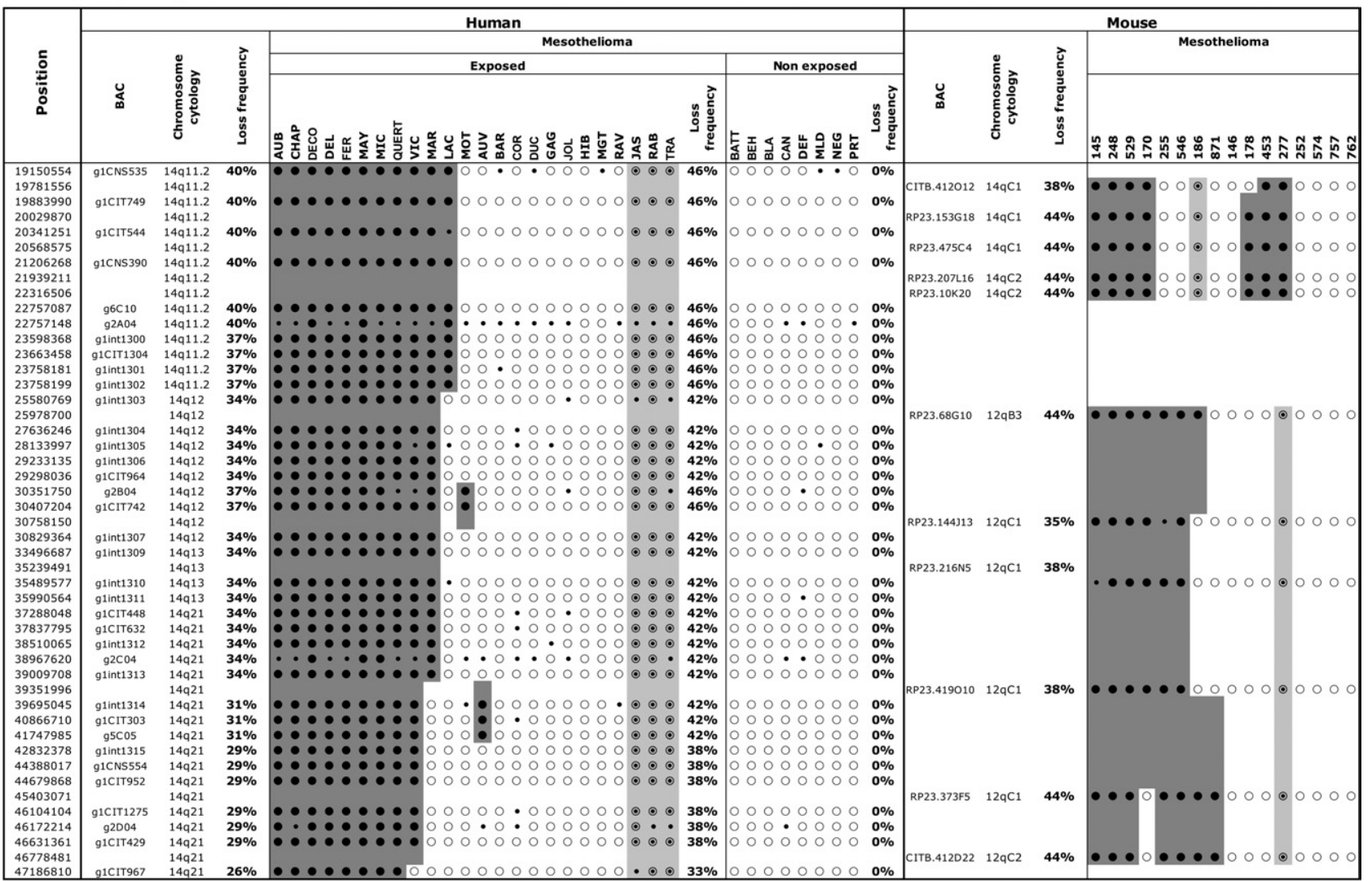

Figure 4. Schematic diagram of chromosomal alterations in the Hs 14q11.2-q21 region in asbestos-exposed and non-asbestos-exposed cases, and in the syntenic mouse chromosomal regions. The start position in the human genome (NCBI Build 36), the cytogenetic location, and the alteration frequency of each BAC clone are shown in the left columns. Each vertical column represents one individual MM: Open circle, no evidence of loss or gain; large black circle, heterozygous loss; open circle with a central dot, gain; small black circle, not informative; dark gray shaded area, region of loss; light gray shaded area, region of gain. Corresponding mouse chromosomal regions are shown when alteration frequency of BAC clones is greater than $30 \%$. Alteration frequency of each BAC clone is also specified for each group.

quent in tumorigenic (T) than in nontumorigenic (NT) human $\mathrm{MM}$, corresponding to losses in 3p23-p14 (T: 74\%; NT: 33\%), 1p31-p12 (T: 52\%; NT: 22\%), 10p15-p12 (T: 43\%; NT: 22\%), and 17p13-p11.2 (T: 43\%; NT: 22\%), and gain in 5p15.3-p11 (61\%; NT: 33\%). Statistical comparison between the occurrence of minimal regions of chromosomal alterations (Table 6) and this biological feature revealed that losses of 1p22-p21 ( $P<0.05)$, 3p21 $(P<0.05)$, and 9p22 $(P<0.02)$ regions were more frequent in tumorigenic than in nontumorigenic MM. Interestingly, the $3 p 21$ region contained several genes previously described as being involved in tumor progression (SETD2, MST1R, PLXNB1, RASSF1, RBM5, SEMA3B, SEMA3F, HYAL 1, and HYAL2).

The number of breakpoints across the 22 autosomes was quantified to estimate genomic instability in human MM. Breakpoints were defined as the junction between a nonaltered chromosomal region and a lost region. The breakpoint index, which corresponds to the sum of all breakpoints observed, ranged from 2 to 35, with a median of 22.5 (Figure 3). Interestingly, a statistically significant correlation was demonstrated between a high breakpoint index and the ability of MMs to develop a tumor in nude mice $(P=0.031)$.

\section{Comparative Genomic Alterations Related to Fiber Exposure}

Comparison of recurrent regions of chromosomal alterations between asbestos-exposed (24 cases) and nonasbestos-exposed (8 cases) patients showed a difference in the 14q11.2-q21 region (Figure 4). The maximum frequency of chromosomal alterations was $46 \%$ and $0 \%$ in asbestos-exposed and non-asbestos-exposed cases, respectively $(P<0.03)$. In our murine model of fiberinduced $\mathrm{MM}$, the syntenic regions 12qB3-qC1 and $14 q C 1-q C 2$ were also altered at a frequency of $44 \%$ (Table 5).

\section{Discussion}

This study was designed to compare genomic alterations in human MMs and in fiber-related murine MMs as models of human MM. An aCGH study of mineral fiber-induced murine MM was recently reported by Altomare et al (2009). ${ }^{36}$ This investigation concerned four MMs developed in $\mathrm{Arf}^{+/-}$mice and primarily identified losses or gains of whole chromosomes, but the only recurrent genomic imbalance was a focal loss in chromosome 4C6 
containing the Cdkn2a locus and the Faf1 gene. ${ }^{36}$ The present study, based on 16 murine MMs, demonstrated numerous genomic alterations involving several chromosomes. Similarly, two minimal regions of chromosomal loss were identified, containing Cdkn2a/Cdkn2b, and Cdkn2c and Faf1, respectively. In addition to recurrent deletions in mouse chromosome 4, frequent losses included regions in chromosomes 7 and 14, and frequent gains were detected in chromosomes 6, 8, 15, and 19, likely because of the greater number of MMs investigated in this series. Another aCGH study was carried out in 11 cases of iron saccharate-induced MM (six epithelioid and five sarcomatoid) in rats. ${ }^{37}$ Epithelioid MMs showed minimal alterations, whereas a few chromosomal amplifications and deletions were found in sarcomatoid MM, including homozygous deletion of the Cdkn2a/Cdkn2b locus. Further studies would be necessary to determine whether these differences are related to the type of MMinducing agent and/or to species specificities. However, Cdkn2a/Cdkn2b locus deletion seems to be an independent and specific feature of MM.

Human MMs have been previously studied by various cytogenetic methods: classical CGH, CGH array, single nucleotide polymorphism array, and representational oligonucleotide microarray analysis. Human MM is characterized by frequent losses of chromosomes $1 p, 3 p, 4,6$, $9 p, 13 q, 14 q$, and $22 q$ and by gains mostly involving chromosomes $5 p, 7 p, 8 q$, and $17 q .{ }^{21-26,45,46}$ In agreement with data in the literature, aCGH in our series detected chromosomal losses in similar regions, confirming the recurrence of these alterations in human MM. Other regions of loss identified in 8p, 10p, 14q, 15q, 17p, 18q, $19 p$, and $19 q$ have also been reported in some studies. ${ }^{47,48}$ In the $12 p$ region, a translocation breakpoint with $X$ chromosome was described in one human MM. ${ }^{49}$ In the present study, recurrent regions of gain were identified only in $5 p, 7 p$, and $20 q$. Data in the literature tend to indicate that MM tumors show more marked diversity of chromosomal gains.

Genomic data obtained with cultured MM cells are in good agreement with those found in MM primary tumors; ie, all recurrent regions of chromosomal alterations identified in cultures of human MM, except for losses in 8p23p12 and 12p13, have been previously described in primary tumors using $\mathrm{CGH}, \mathrm{CGH}$ array, or representational oligonucleotide microarray analysis (see Supplementary Table S1 at http://ajp.amjpathol.org). Nevertheless, a higher frequency of alterations was observed in MM cultured cells, probably because of contamination of tumor specimens by normal tissue.

Similarities and differences were clearly demonstrated after "humanizing" murine MM genomic profiles. The most striking similarity between human disease and its murine model is the deletion of CDKN2A/CDKN2B locus, with a frequency as high as $91 \%$ and $81 \%$ in humans and mice, respectively, and this deletion is frequently biallelic in both species (Table 5). These results are in agreement with published data reporting that CDKN2A/CDKN2B deletions are a feature of MM. ${ }^{28,29,50,51}$ The present results suggest codeletion of CDKN2A and MTAP (methylthioadenosine phosphorylase), a gene under study as a po- tential therapeutic target. ${ }^{51,52}$ Interestingly, the orthologous Mtap gene is located in the syntenic region $(\mathrm{Mm}$ chr4C4), and could be accordingly codeleted with Cdkn2a in the murine genome.

Loss of Hs 1p36.3-p35 (Mm 4qD2.3-4qE2) is another notable similarity. Deletions in the short arm of chromosome 1 are a well-known feature of human $\mathrm{MM}^{53,54}$ and were associated with high asbestos exposure level in one study. ${ }^{55}$ In our series, losses of $1 \mathrm{p} 36.3$ were found in 51\% and $63 \%$ of cases in human and murine MM, respectively (Table 5). One gene, CHD5, may be of interest in this region. CHD5 is involved in chromatin remodeling, and has been defined as a tumor suppressor gene. ${ }^{56}$ Monoallelic loss of this gene was observed in several human MMs in the present series. Other studies reported that $\mathrm{CDH} 5$ inactivation was associated with silencing of the second allele by an epigenetic mechanism, as in neuroblastoma and several carcinomas. ${ }^{57,58}$

The minimal region of common deletion between $\mathrm{Hs}$ 13q14-q33 and Mm 14qD1-qE2.3 was found at a frequency of $58 \%$ and $69 \%$, respectively. The minimal region of deletion, 13q21, contains only three genes, $P C D H 9, K L H L 1$, and $D A C H 1$. Interestingly, a biallelic deletion was found in this region in one human MM. PCDH9 is a member of the protocadherin family, involved in oncogenesis. ${ }^{59} \mathrm{PCDH9}$ was found to be mutated in one pancreatic primary tumor ${ }^{60}$ and $D A C H 1$ is a nuclear factor playing a role in breast, prostate, and ovary cancers. ${ }^{61}$

The 5p15.3-p13 gains reported in human MM were found in different syntenic regions of the mouse genome (Table 5). Many genes of interest are located in these regions, including several cadherins, PRMD9, SKP2, and RAD1. TERT is located in $5 p 15.3$, a recurrent region of gain in human MM but not in the syntenic mouse region. In one human MM, 5p15.3 was also amplified. Telomerase activity has been reported in a high proportion of human pleural $\mathrm{MM}$, and telomere maintenance was recently reported to be due to both telomerase activity and alternative lengthening of telomeres in peritoneal MM. ${ }^{62,63}$ Tert appears to be constitutively active in most murine cells, ${ }^{64}$ and the $\mathrm{Mm} \mathrm{13qC1}$ region in which it is located is not deleted in murine MM, in accordance with a potential role of this enzyme in both species.

In contrast, several regions of genomic alterations differed between human and murine MMs. In the present series, a high rate of deletions was detected in $\mathrm{Hs} 22 \mathrm{q}$ mainly consisting of loss of the entire chromosome, and NF2 inactivation is a specific alteration in MM. ${ }^{18,27,53,55}$ Monoallelic deletions at NF2 were found in $71 \%$ of cases, and a biallelic deletion was observed in one MM. Lower percentages were observed in murine $\mathrm{MM}$ genomic profiles. However, genetic analyses confirmed loss of $N f 2$ in murine MM, in agreement with a role of this gene in mesothelial carcinogenesis (Table 7). The Nf2 gene has been shown to enhance the incidence of $\mathrm{MM}$ in mice without shortening the latency, in comparison with WT mice, supporting a role as a susceptibility factor, a hypothesis previously formulated in human MM. ${ }^{65}$ Furthermore, MM occurred at a higher incidence when mice knockout for Nf2 were co-inactivated for either Ink4a/Arf 
or Trp53. ${ }^{66}$ These observations indicate that inactivation of other genes potentiates Nf2 gene loss and substantially contributes to development of MM. Other genes located in Hs 22q could also play a role in mesothelial carcinogenesis, such as EP30O and BIK located in the region lost at the highest frequency. Further studies should be developed to identify other genes relevant to mesothelial oncogenesis.

Deletions of $3 p$ regions are among the most frequent alterations in human cancers, including human MM. Frequent loss of the Hs 3p21 region, in which several candidate genes are located, was observed, but not in mice. A cluster of tumor suppressor genes has been reported in the 3p21.3 region, including Ras-associated factor 1 (RASSF1), which appears to be inactivated by methylation in MM. ${ }^{67-69}$ Alterations in this $3 p 21$ region were significantly more frequent in human $\mathrm{MM}$ exhibiting tumorigenicity in nude mice than in nontumorigenic MM, suggesting an important role of the associated genes in tumor formation. However, as for chromosome 22, the syntenic region of human 3p21 was not altered accordingly in mice. These differences could be explained by technical issues, including differences in resolution of human and murine arrays used for genomic profiling, as well as species specificities.

Other regions of chromosome imbalance found exclusively in human MM contain well-known TSG such as BRCA2 (13q13, 60\% frequency of loss) or PTEN (10q23$24,37 \%$ frequency of loss). Downregulation of PTEN has been previously described in human $\mathrm{MM}^{70}$ In contrast, some recurrent regions of chromosomal alterations did not contain any potential TSG or oncogene, but encompassed loci of several miRNAs. Differential expression of miRNAs has been previously observed between mesothelioma and mesothelial cells. ${ }^{71,72}$ Copy number alteration is one of the mechanisms affecting miRNAs gene expression. MiRNAs are thought to act as TSGs and oncogenes because of their ability to modulate the transcriptional regulation of their target genes. Indeed, MIR31, located in the 9p21 recurrently lost region (Table 6 ), was recently demonstrated to inhibit cell proliferation and invasion of mesothelioma cells. ${ }^{73}$ Further investigations of other miRNAs, such as MIR760, MIR137, and MIR553, would be of interest. These miRNAs are located in 1p22-21, a region devoid of coding genes, but altered at a higher frequency in tumorigenic MM than in nontumorigenic human MM. The present study identified several miRNAs that could be deregulated in both human and murine MMs.

Comparison of recurrent regions of chromosomal alterations according to MM histology showed different frequencies between epithelioid and sarcomatoid subtypes. Although the differences are not significant, at least partly because of the low power of the statistical analysis, they are likely species dependent. This suggests that recurrent regions of chromosomal alterations are characteristic of a given subtype only in a given species. Differences in the proportion of histological subtypes between human and mouse complicated comparison between these two species. Several authors have emphasized that epithelioid MM was more frequent in humans than in mice after asbestos exposure. ${ }^{31,32}$ More recently, Jongsma et $\mathrm{al}^{66}$ found the same result in spontaneous MM identified in mice carrying conditional TSG knockout alleles for Nf2, Trp53, and Ink4a/Arf. In this study, eight different series of mice carrying co-inactivation of two or three TSGs were studied. The proportion of different subtypes was dependent on the genetic background, with epithelioid MM being under-represented in all but one series. These authors suggested that the predominant epithelioid MM subtype seen in humans as opposed to mice may occur as a result of species-specific differences or relate to the route of MM induction. Our results would better suggest a species-specific difference.

An interesting finding was the relationship between chromosomal instability, estimated by the number of breakpoints, and the ability of human MM xenografts to develop subcutaneous tumors in nude mice. To the best of our knowledge, this relationship with tumor aggressiveness has not been previously reported in mesothelioma. However, in other types of cancer, an increase in chromosomal aberrations is associated with higher grade and stage of tumors, which is consistent with increased genomic instability observed in tumor development and progression. ${ }^{74,75}$ It would be of interest to determine whether the number of breakpoints could be a useful prognostic factor for MM progression.

The present study identified one recurrent region of chromosome loss, 14q11.2-q21, in asbestos-exposed patients that was not found in non-asbestos-exposed patients but that also was lost in the syntenic region in murine mesotheliomas, suggesting that this region might be a target of action of mineral fibers. Frequent allelic loss has been detected in this region in human MM. ${ }^{21,76}$ However, to date, it has been related to asbestos exposure in only one study by Björkqvist et al, ${ }^{76}$ who found a clear history of asbestos exposure in nine of $13 \mathrm{MMs}$ with deletions at 14q, compared with only one of five MMs with no known exposure to asbestos. Several genes involved in cell cycle regulation (CCNB1IP1), DNA repair (PARP2), and tumor promoting transcription factor (NKX2-1) are located in this region. Moreover, Sugarbaker et al $(2008)^{77}$ identified $\mathrm{LOH}$ in the $14 \mathrm{q} 11$ region involving LRP10, a member of low-density lipoprotein receptorrelated proteins, in one patient with a history of asbestos exposure.

In conclusion, despite certain differences, our results show common genomic alterations between genomic profiles in murine and human $\mathrm{MM}$, and suggest that these alterations can be of importance to account for asbestosinduced mesothelial cell neoplastic transformation. Genes at the CDKN2A/CDKN2B locus may play an important role in this process by committing cells to carcinogenic transformation. It would be of interested to determine whether other genes located in common regions of chromosomal imbalance in these two species could be involved in mesothelioma transformation. Genomic profiling of human and murine MM cells reported here validates the murine model as a useful model to study the molecular mechanisms of this disease and to test innovative therapeutic approaches. New generations of 
genomic profiling tools in combination with expression analysis will be useful to identify target genes involved in this dreadful disease.

\section{Acknowledgments}

Murine aCGH was performed at the CIT platform at Institut Curie by Camille Blanchard. Human aCGH was performed at the CIT platform at IGBMC. We thank A. Janin for her participation in histological examinations. We are grateful to clinicians and histologists for having provided tissue samples: Dr. Isame Abd-Alsamad and Prof. JeanClaude Pairon (CHI, Créteil, France), Françoise Le PimpecBarthes and Claire Danel and Prof. Marc Riquet (HEGP, Paris, France), and Philippe Astoul and Christian Boutin (Hôpital la Conception and CHU, Marseille, France).

\section{References}

1. Robinson BW, Lake RA: Advances in malignant mesothelioma. N Eng J Med 2005, 353:1591-1603

2. Goldberg M, Imbernon E, Rolland P, Gilg Soit Ilg A, Saves M, de Quillacq A, Frenay C, Chamming S, Arveux P, Boutin C, Launoy G, Pairon JC, Astoul P, Galateau-Salle F, Brochard P: The French National Mesothelioma Surveillance Program. Occup Environ Med 2006, 63:390-395

3. Mowe G, Gylseth B: Occupational exposure and regional variation of malignant mesothelioma in Norway, 1970-79. Am J Ind Med 1986, 9:323-332

4. Albin M, Magnani C, Krstev S, Rapiti E, Shefer I: Asbestos and cancer: an overview of current trends in Europe. Environ Health Perspect 1999, 107(Suppl 2):289-298

5. Kishimoto T, Ozaki S, Kato K, Nishi H, Genba K: Malignant pleural mesothelioma in parts of Japan in relationship to asbestos exposure. Ind Health 2004, 42:435-439

6. Park EK, Hannaford-Turner KM, Hyland RA, Johnson AR, Yates DH: Asbestos-related occupational lung diseases in NSW. Australia and potential exposure of the general population. Ind Health 2008, 46: 535-540

7. Pan XL, Day HW, Wang W, Beckett LA, Schenker MB: Residential proximity to naturally occurring asbestos and mesothelioma risk in California. Am J Respir Crit Care Med 2005, 172:1019-1025

8. Tward JD, Wendland MM, Shrieve DC, Szabo A, Gaffney DK: The risk of secondary malignancies over 30 years after the treatment of nonHodgkin lymphoma. Cancer 2006, 107:108-115

9. Hodgson DC, Gilbert ES, Dores GM, Schonfeld SJ, Lynch CF, Storm $\mathrm{H}$, Hall P, Langmark F, Pukkala E, Andersson M, Kaijser M, Joensuu H, Fossa SD, Travis LB: Long-term solid cancer risk among 5-year survivors of Hodgkin's Iymphoma. J Clin Oncol 2007, 25:1489-1497

10. Goodman JE, Nascarella MA, Valberg PA: Ionizing radiation: a risk factor for mesothelioma. Cancer Causes Control 2009, 20:1237-1254

11. Goldberg S, Rey G, Luce D, Gilg Soit Ilg A, Rolland P, Brochard P, Imbernon E, Goldberg M: Possible effect of environmental exposure to asbestos on geographical variation in mesothelioma rates. Occup Environ Med 2010, 67:417-421

12. Ryman-Rasmussen JP, Tewksbury EW, Moss OR, Cesta MF, Wong BA, Bonner JC: Inhaled multiwalled carbon nanotubes potentiate airway fibrosis in murine allergic asthma. Am J Respir Cell Mol Biol 2009, 40:349-358

13. Donaldson K, Murphy FA, Duffin R, Poland CA: Asbestos, carbon nanotubes and the pleural mesothelium: a review and the hypothesis regarding the role of long fibre retention in the parietal pleura, inflammation and mesothelioma. Part Fibre Toxicol 2010, 7:5

14. Poland CA, Duffin R, Kinloch I, Maynard A, Wallace WA, Seaton A, Stone V, Brown S, Macnee W, Donaldson K: Carbon nanotubes introduced into the abdominal cavity of mice show asbestos-like pathogenicity in a pilot study. Nat Nanotechnol 2008, 3:423-428

15. Takagi A, Hirose A, Nishimura T, Fukumori N, Ogata A, Ohashi N, Kitajima S, Kanno J: Induction of mesothelioma in p $53+/-$ mouse by intraperitoneal application of multi-wall carbon nanotube. J Toxicol Sci 2008, 33:105-116

16. Sakamoto Y, Nakae D, Fukumori N, Tayama K, Maekawa A, Imai K, Hirose A, Nishimura T, Ohashi N, Ogata A: Induction of mesothelioma by a single intrascrotal administration of multi-wall carbon nanotube in intact male Fischer 344 rats. J Toxicol Sci 2009, 34:65-76

17. Gibas Z, Li FP, Antman KH, Bernal S, Stahel R, Sandberg AA: Chromosome changes in malignant mesothelioma. Cancer Genet Cytogenet 1986, 20:191-201

18. Hagemeijer A, Versnel MA, Van Drunen E, Moret M, Bouts MJ, Van der Kwast TH, Hoogsteden HC: Cytogenetic analysis of malignant mesothelioma. Cancer Genet Cytogenet 1990, 47:1-28

19. Kivipensas P, Bjorkqvist AM, Karhu R, Pelin K, Linnainmaa K, Tammilehto L, Mattson K, Kallioniemi QP, Knuutila S: Gains and losses of DNA sequences in malignant mesothelioma by comparative genomic hybridization. Cancer Genet Cytogenet 1996, 89:7-13

20. De Rienzo A, Balsara BR, Apostolou S, Jhanwar SC, Testa JR: Loss of heterozygosity analysis defines a $3-\mathrm{cM}$ region of $15 \mathrm{q}$ commonly deleted in human malignant mesothelioma. Oncogene 2001, 20 : 6245-6249

21. Krisman M, Müller KM, Jaworska M, Johnen G: Molecular cytogenetic differences between histological subtypes of malignant mesotheliomas: DNA cytometry and comparative genomic hybridization of 90 cases J Pathol 2002, 197:363-371

22. Lindholm PM, Salmenkivi K, Vauhkonen H, Nicholson AG, Anttila S, Kinnula VL, Knuutila S: Gene copy number analysis in malignant pleural mesothelioma using oligonucleotide array CGH. Cytogenet Genome Res 2007, 119:46-52

23. Taniguchi T, Karnan S, Fukui T, Yokoyama T, Tagawa H, Yokoi K, Ueda Y, Mitsudomi T, Horio Y, Hida T, Yatabe Y, Seto M, Sekido Y Genomic profiling of malignant pleural mesothelioma with arraybased comparative genomic hybridization shows frequent non-random chromosomal alteration regions including JUN amplification on 1p32. Cancer Sci 2007, 98:438-446

24. Ivanov SV, Miller J, Lucito R, Tang C, Ivanova AV, Pei J, Carbone M, Cruz C, Beck A, Webb C, Nonaka D, Testa JR, Pass HI: Genomic events associated with progression of pleural malignant mesothelioma. Int J Cancer 2009, 124:589-599

25. Cheung M, Pei J, Pei Y, Jhanwar SC, Pass HI, Testa JR: The promyelocytic leukemia zinc-finger gene, PLZF, is frequently downregulated in malignant mesothelioma cells and contributes to cell survival. Oncogene 2010, 29:1633-1640

26. Christensen BC, Houseman EA, Poage GM, Godleski JJ, Bueno R, Sugarbaker DJ, Wiencke JK, Nelson HH, Marsit CJ, Kelsey KT: Integrated profiling reveals a global correlation between epigenetic and genetic alterations in mesothelioma. Cancer Res 2010, 70:56865694

27. Sekido Y, Pass HI, Bader S, Mew DJ, Christmas MF, Gazdar AF Neurofibromatosis type 2 (NF2) gene is somatically mutated in mesothelioma but not in lung cancer. Cancer Res 1995, 55:1227-1231

28. Xiao S, Li DZ, Vijg J, Sugarbaker DJ, Corson JM, Fletcher JA: Codeletion of p15 and p16 in primary malignant mesothelioma. Oncogene 1995, 11:511-515

29. Hirao T, Bueno R, Chen CJ, Gordon GJ, Heilig E, Kelsey KT: Alterations of the p16INK4 locus in human malignant mesothelial tumors. Carcinogenesis 2002, 23:1127-1130

30. Kitamura F, Araki S, Suzuki Y, Yokoyama K, Tanigawa T, Iwasaki R: Assessment of the mutations of p53 suppressor gene and $\mathrm{Ha}$ - and Ki-ras oncogenes in malignant mesothelioma in relation to asbestos exposure: a study of 12 American patients. Ind Health 2002, 40:175181

31. Fleury-Feith J, Lecomte C, Renier A, Matrat M, Kheuang L, Abramowski V, Levy F, Janin A, Giovannini M, Jaurand MC: Hemizygosity of $\mathrm{N} f 2$ is associated with increased susceptibility to asbestosinduced peritoneal tumours. Oncogene 2003, 22:3799-3805

32. Altomare DA, Vaslet CA, Skele KL, De Rienzo A, Devarajan K, Jhanwar SC, McClatchey AI, Kane AB, Testa JR: A mouse model recapitulating molecular features of human mesothelioma. Cancer Res 2005, 65:8090-8095

33. Kane AB: Animal models of malignant mesothelioma. Inhal Toxicol 2006, 18:1001-1004

34. Lecomte C, Andujar P, Renier A, Kheuang L, Abramowski V, Mellottee L, Fleury-Feith J, Zucman-Rossi J, Giovannini M, Jaurand MC: Similar 
tumor suppressor gene alteration profiles in asbestos-induced murine and human mesothelioma. Cell Cycle 2005, 4:1862-1869

35. Andujar P, Lecomte C, Renier A, Fleury-Feith J, Kheuang L, Daubriac $J$, Janin A, Jaurand MC: Clinico-pathological features and somatic gene alterations in refractory ceramic fibre-induced murine mesothelioma reveal mineral fibre-induced mesothelioma identities. Carcinogenesis 2007, 28:1599-1605

36. Altomare DA, Menges CW, Pei J, Zhang L, Skele-Stump KL, Carbone M, Kane AB, Testa JR: Activated TNF-alpha/NF-kappaB signaling via down-regulation of Fas-associated factor 1 in asbestos-induced mesotheliomas from Arf knockout mice. Proc Natl Acad Sci U S A 2009 , 106:3420-3425

37. Hu Q, Akatsuka S, Yamashita Y, Ohara H, Nagai H, Okazaki Y, Takahashi T, Toyokuni S: Homozygous deletion of CDKN2A/2B is a hallmark of iron-induced high-grade rat mesothelioma. Lab Invest 2010, 90:360-373

38. Pairon JC, Orlowski E, Iwatsubo Y, Billongalland MA, Dufour G, Chammings S, Archambault C, Bignon J, Brochard P: Pleural mesothelioma and exposure to asbestos-evaluation from work histories and analysis of asbestos bodies in bronchoalveolar lavage fluid or lung tissue in 131 patients. Occup Environ Med 1994, 51:244-249

39. dos Santos NR, Rickman DS, de Reynies A, Cormier F, Williame M, Blanchard C, Stern MH, Ghysdael J: Pre-TCR expression cooperates with TEL-JAK2 to transform immature thymocytes and induce T-cell leukemia. Blood 2007, 109:3972-3981

40. Mariani O, Brennetot C, Coindre JM, Gruel N, Ganem C, Delattre O, Stern $\mathrm{MH}$, Aurias A: JUN oncogene amplification and overexpression block adipocytic differentiation in highly aggressive sarcomas. Cancer Cell 2007, 11:361-374

41. Neuvial P, Hupe P, Brito I, Liva S, Manie E, Brennetot C, Radvanyi F, Aurias A, Barillot E: Spatial normalization of array-CGH data. BMC Bioinformatics 2006, 7:264

42. La Rosa P, Viara E, Hupe P, Pierron G, Liva S, Neuvial P, Brito I, Lair S, Servant N, Robine N, Manie E, Brennetot C, Janoueix-Lerosey I, Raynal V, Gruel N, Rouveirol C, Stransky N, Stern MH, Delattre O, Aurias A, Radvanyi F, Barillot E: VAMP: visualization and analysis of array-CGH, transcriptome and other molecular profiles. Bioinformatics 2006, 22:2066-2073

43. Hupe P, Stransky N, Thiery JP, Radvanyi F, Barillot E: Analysis of array CGH data: from signal ratio to gain and loss of DNA regions. Bioinformatics 2004, 20:3413-3422

44. Vivo C, Liu J, Broaddus C: c-jun N-terminal kinase contributes to apoptotic synergy induced by tumor necrosis factor-related apoptosis-inducing ligand plus DNA damage in chemoresistant, p53 inactive mesothelioma cells. J Biol Chem 2003, 278:25461-25467

45. Knuutila S, Armengol G, Bjorkqvist AM, el-Rifai W, Larramendy ML, Monni O, Szymanska J: Comparative genomic hybridization study on pooled DNAs from tumors of one clinical-pathological entity. Cancer Genet Cytogenet 1998, 100:25-30

46. Balsara BR, Bell DW, Sonoda G, De Rienzo A, du Manoir S, Jhanwar SC, Testa JR: Comparative genomic hybridization and loss of heterozygosity analyses identify a common region of deletion at 15q11.1-15 in human malignant mesothelioma. Cancer Res 1999, 59:450-454

47. Sandberg AA, Bridge JA: Updates on the cytogenetics and molecular genetics of bone and soft tissue tumors. Mesothelioma Cancer Genet Cytogenet 2001, 127:93-110

48. Gray SG, Fennell DA, Mutti L, O'Byrne KJ: In arrayed ranks: array technology in the study of mesothelioma. J Thorac Oncol 2009, 4:411-425

49. Aerssens J, Guo C, Vermeesch J, Baens M, Browne D, Litt M, Van Den Berghe $\mathrm{H}$, Marynen $\mathrm{P}$ : A physical map of the region spanning the chromosome 12 translocation breakpoint in a mesothelioma with a $\mathrm{t}(\mathrm{X} ; 12)(\mathrm{q} 22 ; \mathrm{p} 13)$. Cytogenet Cell Genet 1995, 71:268-275

50. Prins JB, Williamson KA, Kamp MM, Van Hezik EJ, Van der Kwast TH, Hagemeijer A, Versnel MA: The gene for the cyclin-dependent-kinase-4 inhibitor, CDKN2A, is preferentially deleted in malignant mesothelioma. Int J Cancer 1998, 75:649-653

51. Ladanyi M: Implications of P16/CDKN2A deletion in pleural mesotheliomas. Lung Cancer 2005, 49(Suppl 1):S95-S98

52. Kindler HL, Burris HA 3rd, Sandler AB, Oliff IA: A phase II multicenter study of L-alanosine, a potent inhibitor of adenine biosynthesis, in patients with MTAP-deficient cancer. Invest New Drugs 2009, 27 : $75-81$
53. Taguchi T, Jhanwar SC, Siegfried JM, Keller SM, Testa JR: Recurrent deletions of specific chromosomal sites in 1p, 3p, 6q, and 9p in human malignant mesothelioma. Cancer Res 1993, 53:4349-4355

54. Lee WC, Testa JR: Somatic genetic alterations in human malignant mesothelioma (review). Int J Cancer 1999, 14:181-188

55. Tiainen M, Tammilehto L, Rautonen J, Tuomi T, Mattson K, Knuutila S: Chromosomal abnormalities and their correlations with asbestos exposure and survival in patients with mesothelioma. Br J Cancer 1989 60:618-626

56. Bagchi A, Papazoglu C, Wu Y, Capurso D, Brodt M, Francis D, Bredel M, Vogel H, Mills AA: CHD5 is a tumor suppressor at human $1 \mathrm{p} 36$. Cell 2007, 128:459-475

57. Fujita T, Igarashi J, Okawa ER, Gotoh T, Manne J, Kolla V, Kim J, Zhao H, Pawel BR, London WB, Maris JM, White PS, Brodeur GM: CHD5, a tumor suppressor gene deleted from 1p36.31 in neuroblastomas. J Natl Cancer Inst 2008, 100:940-949

58. Mulero-Navarro S, Esteller M: Chromatin remodeling factor CHD5 is silenced by promoter $\mathrm{CpG}$ island hypermethylation in human cancer. Epigenetics 2008, 3:210-215

59. Berx G, van Roy F: Involvement of members of the cadherin superfamily in cancer. Cold Spring Harb Perspect Biol 2009, 1:a003129

60. Jones S, Zhang X, Parsons DW, Lin JC, Leary RJ, Angenendt P, Mankoo P, Carter H, Kamiyama H, Jimeno A, Hong SM, Fu B, Lin MT, Calhoun ES, Kamiyama M, Walter K, Nikolskaya T, Nikolsky Y, Hartigan J, Smith DR, Hidalgo M, Leach SD, Klein AP, Jaffee EM, Goggins M, Maitra A, Iacobuzio-Donahue C, Eshleman JR, Kern SE, Hruban $\mathrm{RH}$, Karchin R, Papadopoulos N, Parmigiani G, Vogelstein B, Velculescu VE, Kinzler KW: Core signaling pathways in human pancreatic cancers revealed by global genomic analyses. Science 2008 , 321:1801-1806

61. Popov VM, Wu K, Zhou J, Powell MJ, Mardon G, Wang C, Pestell RG: The Dachshund gene in development and hormone-responsive tumorigenesis. Trends Endocrinol Metab 2010, 21:41-49

62. Kumaki F, Kawai T, Churg A, Galateau-Salle FB, Hasleton P, Henderson D, Roggli V, Travis WD, Cagle PT, Ferrans VJ: Expression of telomerase reverse transcriptase (TERT) in malignant mesotheliomas. Am J Surg Pathol 2002, 26:365-370

63. Villa R, Daidone MG, Motta R, Venturini L, De Marco C, Vannelli A, Kusamura S, Baratti D, Deraco M, Costa A, Reddel RR, Zaffaroni N: Multiple mechanisms of telomere maintenance exist and differentially affect clinical outcome in diffuse malignant peritoneal mesothelioma. Clin Cancer Res 2008, 14:4134-4140

64. Rangarajan A, Weinberg RA: Opinion: comparative biology of mouse versus human cells: modelling human cancer in mice. Nat Rev Cancer 2003, 3:952-959

65. Baser ME, De Rienzo A, Altomare D, Balsara BR, Hedrick NM, Gutmann DH, Pitts LH, Jackler RK, Testa JR: Neurofibromatosis 2 and malignant mesothelioma. Neurology 2002, 59:290-291

66. Jongsma J, van Montfort E, Vooijs M, Zevenhoven J, Krimpenfort P, van der Valk M, van de Vijver M, Berns A: A conditional mouse model for malignant mesothelioma. Cancer Cell 2008, 13:261-271

67. Fischer JR, Ohnmacht U, Rieger N, Zemaitis M, Stoffregen C, Kostrzewa M, Buchholz E, Manegold C, Lahm H: Promoter methylation of RASSF1A, RARbeta and DAPK predict poor prognosis of patients with malignant mesothelioma Lung Cancer 2006, 54:109-116

68. Hesson LB, Cooper WN, Latif F: Evaluation of the 3p21.3 tumoursuppressor gene cluster. Oncogene 2007, 26:7283-7301

69. Christensen BC, Godleski JJ, Marsit CJ, Houseman EA, Lopez-Fagundo CY, Longacker JL, Bueno R, Sugarbaker DJ, Nelson HH, Kelsey KT: Asbestos exposure predicts cell cycle control gene promoter methylation in pleural mesothelioma. Carcinogenesis 2008, 29:1555-1559

70. Opitz I, Soltermann A, Abaecherli M, Hinterberger M, Probst-Hensch $\mathrm{N}$, Stahel R, Moch $\mathrm{H}$, Weder W: PTEN expression is a strong predictor of survival in mesothelioma patients. Eur J Cardiothorac Surg 2008, 33:502-506

71. Guled M, Lahti L, Lindholm PM, Salmenkivi K, Bagwan I, Nicholson AG, Knuutila S: CDKN2A, NF2, and JUN are dysregulated among other genes by miRNAs in malignant mesothelioma-a miRNA microarray analysis. Genes Chromosomes Cancer 2009, 48:615-623

72. Busacca S, Germano S, De Cecco L, Rinaldi M, Comoglio F, Favero F, Murer B, Mutti L, Pierotti M, Gaudino G: MicroRNA signature of malignant mesothelioma with potential diagnostic and prognostic implications. Am J Respir Cell Mol Biol 2010, 42:312-319 
73. Ivanov SV, Goparaju CM, Lopez P, Zavadil J, Toren-Haritan G, Rosenwald S, Hoshen M, Chajut A, Cohen D, Pass HI: Pro-tumorigenic effects of miR-31 loss in mesothelioma. J Biol Chem 2010, 285:22809-22817

74. Blaveri E, Brewer JL, Roydasgupta R, Fridlyand J, DeVries S, Koppie T, Pejavar S, Mehta K, Carroll P, Simko JP, Waldman FM: Bladder cancer stage and outcome by array-based comparative genomic hybridization. Clin Cancer Res 2005, 11:7012-7022

75. Chan MW, Hui AB, Yip SK, Ng CF, Lo KW, Tong JH, Chan AW, Cheung HY, Wong WS, Chan PS, Lai FM, To KF: Progressive increase of genetic alteration in urinary bladder cancer by combined allelo- typing analysis and comparative genomic hybridization. Int $\mathrm{J}$ Oncol 2009, 34:963-970

76. Bjorkqvist AM, Wolf M, Nordling S, Tammilehto L, Knuuttila A, Kere J, Mattson K, Knuutila S: Deletions at $14 \mathrm{q}$ in malignant mesothelioma detected by microsatellite marker analysis. Br J Cancer 1999, 81: 1111-1115

77. Sugarbaker DJ, Richards WG, Gordon GJ, Dong L, De Rienzo A, Maulik G, Glickman JN, Chirieac LR, Hartman ML, Taillon BE, Du L, Bouffard P, Kingsmore SF, Miller NA, Farmer AD, Jensen RV, Gullans SR, Bueno R: Transcriptome sequencing of malignant pleural mesothelioma tumors. Proc Natl Acad Sci U S A: 2008, 105:3521-3526 